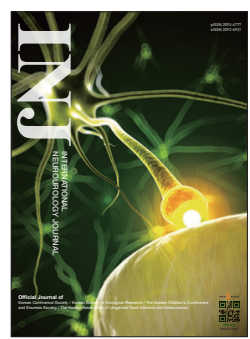

\title{
Seasonal Changes in Lower Urinary Tract Symptoms in Japanese Men With Benign Prostatic Hyperplasia Treated With $\alpha_{1}$-Blockers
}

\author{
Minoru Kobayashi ${ }^{1}$, Akinori Nukui ${ }^{2}$, Takao Kamai ${ }^{3}$ \\ ${ }^{1}$ Department of Urology, Utsunomiya Memorial Hospital, Tochigi, Japan \\ ${ }^{2}$ Department of Urology, Nasu Red Cross Hospital, Tochigi, Japan \\ ${ }^{3}$ Department of Urology, Dokkyo Medical University, Tochigi, Japan
}

Purpose: To investigate whether seasonal changes occurred in lower urinary tract symptoms (LUTS) in patients with benign prostatic hyperplasia (BPH).

Methods: Patients aged 50 years and older with BPH treated with $\alpha_{1}$-blockers were enrolled. The International Prostate Symptom Score (IPSS), quality of life (QoL) score, maximum and average flow rate, voided volume, and postvoid residual volume were measured in summer and winter.

Results: A total of 164 patients were enrolled. The total IPSS and QoL index did not show a significant difference between the 2 seasons. When the IPSS was divided into storage symptoms and voiding symptoms, storage symptoms in winter were substantially but nonsignificantly higher than those in summer $(\mathrm{P}=0.056)$. Of the 7 individual symptoms in the IPSS, a significant seasonal difference was observed only for nocturia, with a higher score in winter. Moreover, none of the uroflowmetric parameters showed a seasonal change. Voided volume had significant correlations with each symptom (urgency and nocturia) and overall subjective scores (storage, total IPSS, and QoL) exclusively in summer, while this correlation remained only for nocturia in winter.

Conclusions: As it has generally been assumed that LUTS deteriorate in winter, the present study corroborated that the severity of storage symptoms was higher in winter than in summer, even in patients treated with $\alpha_{1}$-blockers. In contrast, a seasonal difference was not observed in the uroflowmetric parameters, which may be partly due to the loss of the correlation between subjective and objective measurements of storage symptoms in winter.

Keywords: Adrenergic alpha-Antagonists; Prostatic Hyperplasia; Lower Urinary Tract Symptoms; Seasonal Change; Urodynamics

- Research Ethics: All procedures performed in this study were in accordance with the ethical standards of the Institutional Review Board of Utsunomiya Memorial Hospital (approval number: 26-03). Written informed consent was obtained from all patients.

- Conflict of Interest: No potential conflict of interest relevant to this article was reported.

\section{INTRODUCTION}

It is well recognized that the season of the year exerts an influence on some disease conditions, such as coronary heart dis- ease, cerebrovascular disease, and respiratory disease [1-3]. Additionally, it is generally assumed that lower urinary tract symptoms (LUTS) in patients with benign prostatic hyperplasia (BPH) can be affected by seasonal variation, becoming exacer-

Corresponding author: Minoru Kobayashi (D) https://orcid.org/0000-0002-6859-7064 Department of Urology, Utsunomiya Memorial Hospital, 1-3-16, Ohdori, Utsunomiya city, Tochigi 320-0811, Japan

E-mail: minorukoba@hotmail.com / Tel: +81-28-622-1991 / Fax: +81-28-611-5603

Submitted: February 12, 2017 / Accepted after revision: March 21, 2017 
bated in the cold season. This phenomenon can be explained as follows. Cold stress increases sympathetic activity, which stimulates smooth muscle contraction in the prostate, and induces detrusor overactivity, which decreases the voiding interval and volume $[4,5]$. However, only a few clinical studies have addressed the associations between seasonal changes and LUTS [6-9]. Some studies found significant differences in subjective symptoms between the cold and hot seasons [6,9], while others did not $[7,8]$. Moreover, inconsistent results have been found regarding seasonal variation in some uroflowmetric parameters [7-9]. Such contradictory results prompted us to further investigate the impact of seasonal variation on LUTS. Thus, we conducted a prospective study to assess seasonal changes in urinary symptom scores and uroflowmetric parameters in men with LUTS and BPH.

\section{MATERIALS AND METHODS}

\section{Patients and Study Design}

From January 2014 to August 2016, consecutive patients with $\mathrm{BPH}$ were enrolled in this prospective study. The inclusion criteria were patients aged $\geq 50$ years who had an estimated prostate volume $\geq 20 \mathrm{~mL}$ measured by ultrasonography and had been treated with $\alpha_{1}$-blockers for $\geq 3$ months to avoid the issue of initial treatment efficacy. The exclusion criteria included a repeated history of urinary tract infections, chronic urinary retention, prostate cancer, or any other condition likely to cause urinary problems, as well as the use of antimuscarinics, $\beta 3$ adrenergic agonists, diuretics, or vasopressin. The approval of the Institutional Review Board and informed consent from all patients were obtained beforehand.

\section{Assessment of LUTS}

The International Prostate Symptom Score (IPSS) questionnaire was used to evaluate subjective symptoms. The sum of the storage symptom subscore (frequency, urgency, and nocturia) and the voiding symptom subscore (intermittency, decreased urinary stream, straining, and the feeling of incomplete emptying) was determined, as well as the total score. Uroflowmetry was performed to determine the maximum and average urinary flow rate (Qmax, Qave) and the voided volume (VV). Postvoid residual volume (PVR) was measured by ultrasonography. These measurements were performed once for each patient 6 months apart in the first winter (defined as December, January, and February) and summer (defined as June, July, and August)
Table 1. Monthly average ambient temperatures in the cold and hot seasons from January 2014 to August 2016

\begin{tabular}{lccccccc}
\hline \multirow{2}{*}{ Year } & \multicolumn{3}{c}{ Winter } & & \multicolumn{3}{c}{ Summer } \\
\cline { 2 - 3 } \cline { 6 - 8 } & December & January & February & & June & July & August \\
\hline 2014 & $3.9^{\circ} \mathrm{C}$ & $2.3^{\circ} \mathrm{C}$ & $2.8^{\circ} \mathrm{C}$ & & $21.9^{\circ} \mathrm{C}$ & $25.1^{\circ} \mathrm{C}$ & $26.0^{\circ} \mathrm{C}$ \\
2015 & $7.2^{\circ} \mathrm{C}$ & $3.2^{\circ} \mathrm{C}$ & $3.7^{\circ} \mathrm{C}$ & & $21.4^{\circ} \mathrm{C}$ & $25.6^{\circ} \mathrm{C}$ & $25.4^{\circ} \mathrm{C}$ \\
2016 & - & $3.4^{\circ} \mathrm{C}$ & $4.7^{\circ} \mathrm{C}$ & & $21.7^{\circ} \mathrm{C}$ & $24.2^{\circ} \mathrm{C}$ & $26.2^{\circ} \mathrm{C}$ \\
Average & $5.6^{\circ} \mathrm{C}$ & $3.0^{\circ} \mathrm{C}$ & $3.7^{\circ} \mathrm{C}$ & & $21.7^{\circ} \mathrm{C}$ & $25.0^{\circ} \mathrm{C}$ & $25.9^{\circ} \mathrm{C}$ \\
\hline
\end{tabular}

during the study period to assess seasonal variation. Patients were asked to record the temperature in their bedroom at the time they went to sleep and woke up when they responded to the IPSS questionnaire.

\section{Climate Data}

The average monthly ambient temperatures in degrees Celsius $\left({ }^{\circ} \mathrm{C}\right)$ at the patients' area of residence (longitude $139^{\circ} \mathrm{E}$, latitude $36^{\circ} \mathrm{N}$ ) during the study period were extracted from the Automated Meteorological Data Acquisition System (AMeDAS), operated by the Japan Meteorological Agency, and shown in Table 1.

\section{Statistical Analysis}

Data with a normal distribution were expressed as mean \pm standard deviation. Data with a nonnormal distribution were expressed as median (interquartile range [IQR]). The intragroup difference between the winter and summer values were analyzed by the Wilcoxon rank-sum test. Each pairwise group difference was analyzed using the Mann-Whitney U-test. The Spearman rank correlation coefficient was used to assess correlations between various variables. P-values of $<0.05$ were considered to indicate statistical significance.

\section{RESULTS}

\section{Characteristics of the Patients}

A total of 164 patients, aged $70.9 \pm 7.6$ years, were enrolled in this study. Their mean estimated prostate volume was $34.8 \pm 11.2 \mathrm{~mL}$. The median duration of $\alpha_{1}$-blocker treatment before the study entry was 607 days (IQR, 263-1,128 days). Seventy patients were treated with naftopidil ( $50-75 \mathrm{mg} /$ day), 62 with tamsulosin $(0.2 \mathrm{mg} /$ day), and 32 with silodosin $(8 \mathrm{mg} /$ day). 
Table 2. Comparison of IPSS, QoL score, and uroflometric parameters by season $(\mathrm{n}=164)$

\begin{tabular}{lccc}
\hline Variable & Winter & Summer & P-value \\
\hline IPSS & & & 0.193 \\
Q1: Incomplete emptying & $1(0-2)$ & $1(0-2)$ & 0.081 \\
Q2: Frequency & $1(1-2)$ & $1(1-2)$ & 0.664 \\
Q3: Intermittency & $1(0-2)$ & $1(0-2)$ & 0.976 \\
Q4: Urgency & $1(0-1)$ & $1(0-2)$ & 0.892 \\
Q5: Weak stream & $2(1-3)$ & $2(1-3)$ & 0.472 \\
Q6: Straining & $1(0-2)$ & $1(0-2)$ & 0.018 \\
Q7: Nocturia & $2(1-3)$ & $2(1-2)$ & 0.073 \\
Total score & $10(6-15)$ & $8.5(6-13.8)$ & 0.196 \\
Voiding score & $5(3-8.8)$ & $5(2.3-9)$ & 0.056 \\
Storage score & $4(3-6)$ & $4(3-6)$ & 0.750 \\
QoL score & $3(2-4)$ & $3(2-4)$ & \\
Uroflowmetric parameters & & & 0.383 \\
Qmax (mL/s) & $10.1(7.9-14.0)$ & 0.328 \\
Qave (mL/s) & $5.4(4.0-7.0)$ & $0.0(7.0-15.0)$ & 0.318 \\
VV (mL) & $159.5(109.5-247.3)$ & $5.0(3.5-7.0)$ & \\
PVR (mL) & $45.0(24.3-78.3)$ & $160.0(104.0-254.0)$ & \\
\hline
\end{tabular}

Values are presented as median (interquartile range). P-values were determined by Wilcoxon's rank-sum test.

IPSS, International Prostate Symptom Score; QoL, quality of life; Qmax, maximum urinary flow rate; Qave, average urinary flow rate; VV, voided volume; PVR, postvoid residual.

\section{Seasonal Changes in LUTS}

As shown in Table 2, of the 7 individual symptoms in the IPSS, a significant seasonal difference was observed only for nocturia, which showed a higher score in winter. When the IPSS was divided into storage symptoms and voiding symptoms, the score of storage symptoms in winter was substantially but nonsignificantly higher than in summer $(\mathrm{P}=0.056)$. However, the total IPSS and QoL scores did not show any difference between the 2 seasons. None of the uroflowmetric parameters showed seasonal differences.

Of the 164 participants, 58 provided a complete record of their bedroom temperatures. These patients were divided into 2 groups (high and low bedroom temperature) by the median value of the bedroom temperature. A high bedroom temperature at wake-up time was defined as $\geq 9^{\circ} \mathrm{C}\left(\mathrm{IQR}, 7^{\circ} \mathrm{C}-11.5^{\circ} \mathrm{C}\right)$ in winter and $\geq 26^{\circ} \mathrm{C}$ (IQR, $\left.24^{\circ} \mathrm{C}-27^{\circ} \mathrm{C}\right)$ in summer. The frequency of nocturia was compared between these 2 groups. There was no significant difference in nocturia scores between the 2 groups defined according to their bedroom temperature in either season (Table 3). Likewise, no LUTS other than nocturia were different between the 2 groups (data not shown). Moreover, there were no correlations between bedroom temperature and nocturia score in either season $(r=-0.0241, \mathrm{P}=$ 0.886 for winter, $r=-0.102, \mathrm{P}=0.547$ for summer).
Table 3. Comparison of nocturia score in winter and summer by bedroom temperature

\begin{tabular}{lcc}
\hline Season \& bedroom temperature & Median $(\mathrm{IQR})$ & P-value \\
\hline Winter & & 0.443 \\
High $\left(\geq 9^{\circ} \mathrm{C}\right)(\mathrm{n}=28)$ & $2(1-2)$ & \\
Low $\left(\geq 26^{\circ} \mathrm{C}\right)(\mathrm{n}=34)$ & $2.0(1.3-3.0)$ & \\
Summer & & 0.167 \\
High $\left(\geq 9^{\circ} \mathrm{C}\right)(\mathrm{n}=30)$ & $1.5(1.0-2.8)$ & \\
Low $\left(\geq 26^{\circ} \mathrm{C}\right)(\mathrm{n}=24)$ & $2(2-3)$ & \\
\hline
\end{tabular}

P-values were determined by Mann-Whitney U-test. $\mathrm{IQR}$, interquartile range.

Lastly, in order to explore the degree to which uroflowmetric measurements reflected the subjective symptoms determined by the IPSS in association with seasonal changes, the correlations between Qmax, Qave, VV, and PVR and their corresponding symptoms in the IPSS (i.e., correlations between Qmax/Qave and weak stream, straining, and intermittency; between VV and frequency, urgency, and nocturia; and between PVR and incomplete emptying) as well as the total IPSS and QoL scores were examined in both seasons using the Spearman rank correlation. As shown in Table 4, no symptom score in the IPSS was meaningfully correlated with its uroflowmetric counterpart in either season. However, in summer, VV showed significant negative correlations with overall symptoms, as indicated by storage, to- 


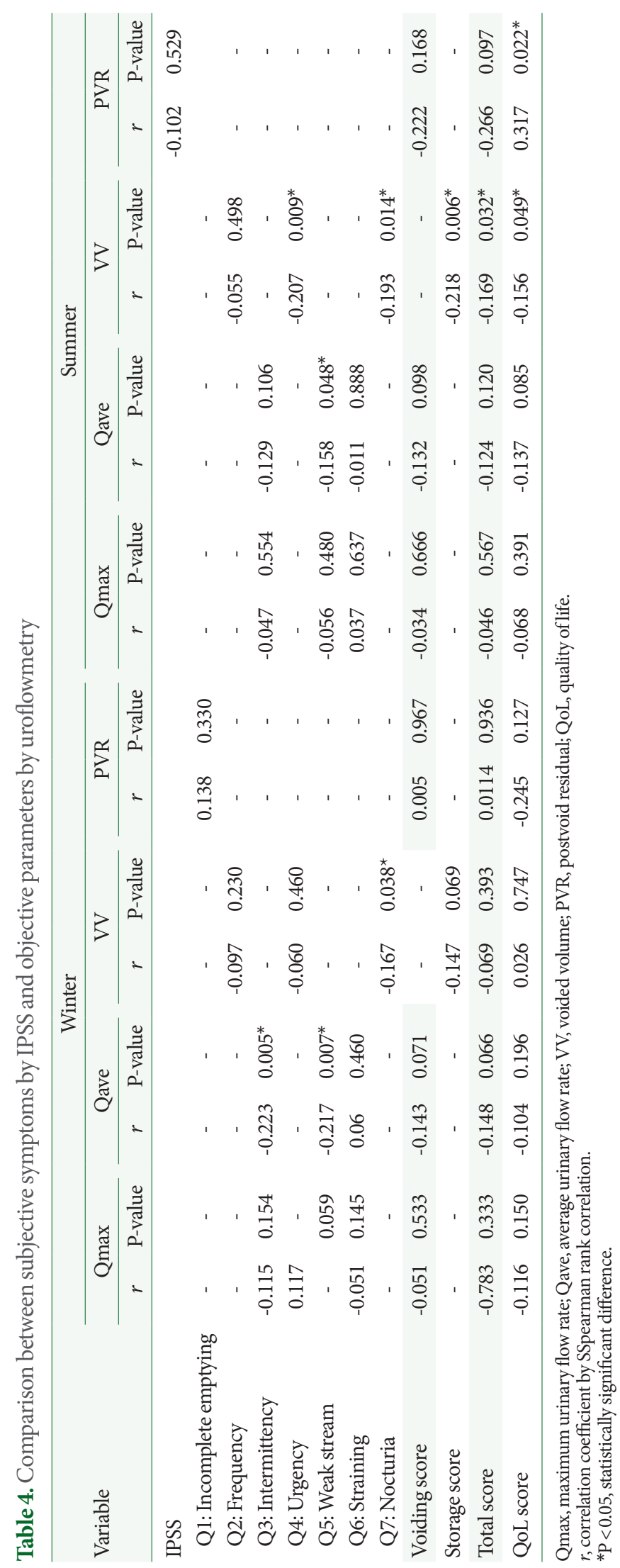

tal IPSS, and QoL scores, as well as with individual storage symptoms, such as urgency and nocturia. In contrast, this correlation was only found with nocturia in winter, suggesting that VV reflected storage symptoms in summer more closely than in winter.

\section{DISCUSSION}

It is well known that cold temperature can induce various physiological responses, such as an increase in blood pressure $[10,11]$, making it possible that cold temperature might affect bladder function, causing LUTS to deteriorate. A questionnaire study showed that feeling colder or warmer is a reason for nighttime frequency in patients with a chief complaint of nocturia [12]. The associations between cold temperature and bladder function have been carefully investigated in animal models, demonstrating that rats stimulated by cold stress showed remarkable detrusor overactivity with increased basal pressure and decreased bladder capacity, which was mediated via $\alpha_{1-}$ adrenoceptors and the resiniferatoxin-sensitive $\mathrm{C}$-fiber nerve pathway $[13,14]$. Changes in body fluid balance may be another factor explaining possible seasonal changes in urinary frequency. That is, reduced diuresis due to insensible fluid loss from sweating in high temperatures results in decreased urinary frequency in the hot season [15]. On the contrary, cold exposure not only provokes peripheral vasoconstriction, which inhibits the secretion of vasopressin and leads to diuresis [16], but also stimulates the secretion of plasma atrial natriuretic peptide, which enhances diuresis [17].

Despite such extensive investigations into the underlying mechanisms and etiology of cold-induced deterioration of storage symptoms, scant evidence supports the association between seasonal changes and LUTS [6-9]. A large community-based questionnaire study conducted among Japanese residents living in 3 different climatic regions demonstrated that storage symptoms, including frequency, urgency, and nocturia, were more severe in winter than in summer [6]. However, this trend was not observed in those living in the area with lower ambient temperatures, which may be due to adaptive responses to cold stress. This observation suggests that seasonal variation in LUTS may be affected by the ambient temperature change throughout a year. That is the only study showing seasonal variation in storage symptoms that was carried out in healthy participants. A small longitudinal study examining seasonal changes in the IPSS and uroflowmetric parameters in 31 Japa- 
nese patients with LUTS treated by medication showed no significant difference in the total IPSS, voiding symptoms, and storage symptoms, but a significant change in Qmax, which was higher in both the cold and comfortable seasons than in the hot season [7]. Another study conducted in the United Kingdom (UK) examined the effect of seasonal variation on the IPSS and uroflowmetric parameters among 296 men with LUTS recruited in 4 different seasons (spring, summer, autumn, and winter) [8]. There were no significant variations in either the IPSS or uroflowmetric parameters by season, with the exception of increased actual nightly voids in frequencyvolume charts over the summer months. A similar retrospective analysis was conducted using a large database of 1,185 Korean men with LUTS, who were categorized into cold-season, hot-season, and intermediate-season groups [9]. The IPSS, especially the scores for storage symptoms and QoL, were significantly aggravated in the cold-season group compared with the hot-season group. Seasonal differences were also observed in some uroflowmetric parameters, with VV being higher and PVR being lower in the cold season than in the hot season. Taken together, although we feel that storage symptoms are likely to become worse in the cold season, this possibility has not always been confirmed in previous studies. However, despite the large number of patients, the 2 recent studies from the UK and Korea [8,9] examined seasonal variation in LUTS not within individuals, but between groups of patients recruited in different seasons, and thus the results were driven by individual differences as well as seasonal differences. With regard to this point, the small study from Japan [7] seems more reliable as an examination of the seasonal variability of LUTS, but the number of patients was too small to draw firm conclusions. Therefore, in order to elucidate the impact of seasonal differences on LUTS, we conducted a prospective study in a larger number of patients, comparing both subjective and objective urinary symptoms measured by the IPSS and uroflowmetry between the cold and hot seasons within the same individuals.

In our study, the severity of nocturia and storage symptoms determined by the IPSS was worse in winter than in summer. Considering our observations from our daily clinical practice and the increasing amount of research into the etiology of cold stress-induced storage symptoms, this finding seems reasonable. When designing our study, we presumed that nighttime frequency might be associated with bedroom temperatures, especially in the cold season, and we hypothesized that lower bedroom temperatures would be correlated with higher night- time frequency. Thus, we examined the difference in nocturia scores between 2 groups classified according to the median bedroom temperature, as well as the correlation between nocturia scores and bedroom temperature. Unexpectedly, we did not find any associations between nighttime frequency and bedroom temperature. This may have been partially due to the smaller number of patients who were available for this analysis, as well as to issues relating to various types of heating and housing. The use of $\alpha_{1}$-blockers may have been another contributor to this negative result, since $\alpha_{1}$-blockers have been shown to potentially inhibit cold stress-induced detrusor overactivity in animal models [13,14].

However, objective measurements by uroflowmetry showed no seasonal variation in our study, although it was assumed that the aggravation of frequency in the cold season would cause a lower VV and flow rate. Nonetheless, some uroflowmetric parameters exhibited even better results in the cold season than in the hot season in the 2 previous studies [7,9]. The authors hypothesized that the higher Qmax in winter was due to a change of temperature caused by movement from the cold outdoors to the warm indoors for measurements, rendering the sympathetic nerve atonic and thus favoring urination [7]. Additionally, it was proposed that the lower VV resulting from decreased diuresis by increased insensible fluid loss in the hot season could lead to a higher VV in the cold season [9]. In any case, there seems to be some discrepancy between the subjective measurements made using the IPSS and the objective measurements by uroflowmetry as a function of the season of the year. Thus, we examined the correlations between uroflowmetric parameters and the IPSS in winter and summer separately. The severity of each symptom in the IPSS did not meaningfully reflect the uroflowmetric measurements in either season. Instead, the overall symptoms indicated by storage, total IPSS, and QoL scores, as well as storage symptoms such as urgency and nocturia, were significantly correlated with VV in the summer, while such a correlation was only found for nocturia in the winter among the same cohort of patients. This suggests that the decreased $\mathrm{VV}$ and flow rate that resulted from the aggravation of storage symptoms in the winter may not have been reflected in uroflowmetric measurements performed in a well-heated examination room. That is, seasonal variations did exist in subjective urinary symptoms, which reflected individuals' daily experiences, but not in objective uroflowmetric parameters that were measured only once in air-conditioned examination rooms, not at the ambient temperature. Several studies have investigated 
the relationship of uroflowmetric parameters and LUTS evaluated by the IPSS, with conflicting results [18-25]. However, no studies have addressed this issue in association with seasonal variations. Discrepancies in the season and ambient temperatures when the studies were conducted may have played a role in producing inconsistent correlations between the IPSS and uroflowmetric variables.

Some limitations of this study need to be acknowledged. First, the number of patients was relatively small. Second, seasonal assessments were performed only once in each patient, and information from the spring and fall was lacking. Third, this was a questionnaire-based study without objective assessments of daytime and nighttime frequency by frequency-volume charts. It is well known that there are discrepancies between nocturia scores on the IPSS and nighttime frequency in frequency-volume charts [26]. In fact, the aforementioned UK study showed a significant increase in median actual nightly voiding episodes over the summer months, despite identical median nocturia scores across the 4 seasons [8]. Lastly, this study was conducted in a limited area in Japan. Therefore, the observations from this study are not applicable to all regions with 4 seasons throughout the world. This point was clearly presented in the aforementioned study conducted in 3 Japanese communities with different ambient temperatures [6].

In conclusion, this study demonstrated that patients with $\mathrm{BPH}$ experienced aggravated storage symptoms in winter compared with summer, even when treated with $\alpha_{1}$-blockers. However, seasonal variation was not observed in the objective variables evaluated by uroflowmetry, which may be partly due to the loss of relationship between subjective symptoms and uroflowmetric measurements, especially in the winter. Therefore, seasonal variations in subjective symptoms should be considered in assessments of the efficacy of therapy for LUTS, especially when initiating or switching drugs [27]. Further research is expected to elucidate the extent and impact of seasonal variations in LUTS for women as well as men across 4 seasons in a larger cohort of patients from more areas with different climate profiles.

\section{REFERENCES}

1. Spencer FA, Goldberg RJ, Becker RC, Gore JM. Seasonal distribution of acute myocardial infarction in the second National Registry of Myocardial Infarction. J Am Coll Cardiol 1998;31:1226-33.

2. Inagawa T, Shibukawa M, Inokuchi F, Tokuda Y, Okada Y, Okada
K. Primary intracerebral and aneurysmal subarachnoid hemorrhage in Izumo City, Japan. Part II: management and surgical outcome. J Neurosurg 2000;93:967-75.

3. Nakaji S, Parodi S, Fontana V, Umeda T, Suzuki K, Sakamoto J, et al. Seasonal changes in mortality rates from main causes of death in Japan (1970--1999). Eur J Epidemiol 2004;19:905-13.

4. Imamura T, Ishizuka O, Nishizawa O. Cold stress induces lower urinary tract symptoms. Int J Urol 2013;20:661-9.

5. Geirsson G, Lindström S, Fall M. The bladder cooling reflex and the use of cooling as stimulus to the lower urinary tract. J Urol 1999;162:1890-6.

6. Yoshimura K, Kamoto T, Tsukamoto T, Oshiro K, Kinukawa N, Ogawa $\mathrm{O}$. Seasonal alterations in nocturia and other storage symptoms in three Japanese communities. Urology 2007;69:864-70.

7. Watanabe T, Maruyama S, Maruyama Y, Kageyama S, Shinbo H, Otsuka A, et al. Seasonal changes in symptom score and uroflowmetry in patients with lower urinary tract symptoms. Scand J Urol Nephrol 2007;41:521-6.

8. Cartwright R, Mariappan P, Turner KJ, Stewart LH, Rajan P. Is there seasonal variation in symptom severity, uroflowmetry and frequency-volume chart parameters in men with lower urinary tract symptoms? Scott Med J 2014;59:162-6.

9. Choi HC, Kwon JK, Lee JY, Han JH, Jung HD, Cho KS. Seasonal variation of urinary symptoms in Korean men with lower urinary tract symptoms and benign prostatic hyperplasia. World J Mens Health 2015;33:81-7.

10. Minami J, Ishimitsu T, Kawano Y, Matsuoka H. Seasonal variations in office and home blood pressures in hypertensive patients treated with antihypertensive drugs. Blood Press Monit 1998:3:101-6.

11. Hanazawa T, Asayama K, Watabe D, Hosaka M, Satoh M, Yasui D, et al. Seasonal variation in self-measured home blood pressure among patients on antihypertensive medications: HOMED-BP study. Hypertens Res 2017;40:284-90.

12. Yoshimura K, Terai A. Fluctuation of night time frequency in patients with symptomatic nocturia. Int J Urol 2005;12:469-73.

13. Imamura $\mathrm{T}$, Ishizuka $\mathrm{O}$, Aizawa $\mathrm{N}$, Zhong $\mathrm{C}$, Ogawa T, Nakayama $\mathrm{T}$, et al. Cold environmental stress induces detrusor overactivity via resiniferatoxin-sensitive nerves in conscious rats. Neurourol Urodyn 2008;27:348-52.

14. Chen Z, Ishizuka O, Imamura T, Aizawa N, Igawa Y, Nishizawa O, et al. Role of alpha1-adrenergic receptors in detrusor overactivity induced by cold stress in conscious rats. Neurourol Urodyn 2009; 28:251-6.

15. Kohn D, Flatau E. Why does cold weather cause frequency of micturition in some elderly people? Br Med J 1980;281:875. 
16. Stocks JM, Taylor NA, Tipton MJ, Greenleaf JE. Human physiological responses to cold exposure. Aviat Space Environ Med 2004;75: 444-57.

17. Hassi J, Rintamäki H, Ruskoaho H, Leppäluoto J, Vuolteenaho O. Plasma levels of endothelin-1 and atrial natriuretic peptide in men during a 2-hour stay in a cold room. Acta Physiol Scand 1991;142: 481-5.

18. Bosch JL, Hop WC, Kirkels WJ, Schröder FH. The International Prostate Symptom Score in a community-based sample of men between 55 and 74 years of age: prevalence and correlation of symptoms with age, prostate volume, flow rate and residual urine volume. Br J Urol 1995;75:622-30.

19. Rosier PF, de Wildt MJ, Wijkstra H, Debruyne FF, de la Rosette JJ. Clinical diagnosis of bladder outlet obstruction in patients with benign prostatic enlargement and lower urinary tract symptoms: development and urodynamic validation of a clinical prostate score for the objective diagnosis of bladder outlet obstruction. J Urol 1996;155:1649-54.

20. van Venrooij GE, Boon TA. The value of symptom score, quality of life score, maximal urinary flow rate, residual volume and prostate size for the diagnosis of obstructive benign prostatic hyperplasia: a urodynamic analysis. J Urol 1996;155:2014-8.

21. Wadie BS, Ibrahim EH, de la Rosette JJ, Gomha MA, Ghoneim MA. The relationship of the International Prostate Symptom Score and objective parameters for diagnosing bladder outlet obstruc- tion. Part I: when statistics fail. J Urol 2001;165:32-4.

22. Porru D, Bartoletti R, Austoni E, Carrino M, Gianneo E, Melloni D. Relationship of flow rate with symptoms, quality of life and other clinical parameters in patients with LUTS suggestive of BPH. Eur Urol 2001;40 Suppl 1:23-7.

23. Vesely S, Knutson T, Damber JE, Dicuio M, Dahlstrand C. Relationship between age, prostate volume, prostate-specific antigen, symptom score and uroflowmetry in men with lower urinary tract symptoms. Scand J Urol Nephrol 2003;37:322-8.

24. Itoh H, Kojima M, Okihara K, Ukimura O, Ushijima S, Kawauchi A, et al. Significant relationship of time-dependent uroflowmetric parameters to lower urinary tract symptoms as measured by the International Prostate Symptom Score. Int J Urol 2006;13:1058-65.

25. Singla S, Garg R, Singla A, Sharma S, Singh J, Sethi P. Experience with uroflowmetry in evaluation of lower urinary tract symptoms in patients with benign prostatic hyperplasia. J Clin Diagn Res 2014;8:NC01-3.

26. van Haarst EP, Bosch JL, Heldeweg EA. The international prostate symptom score overestimates nocturia assessed by frequency-volume charts. J Urol 2012;188:211-5.

27. Morita T, Kubo KK, Fujisaki A, Natsui S, Nukui A, Kobayashi M, et al. Involvement of magnitude of ambient temperature change in nonspecific effect in perceived placebo effect on lower urinary tract symptoms: study on switching of naftopidil in patients with benign prostatic hyperplasia. Res Rep Urol 2013;5:83-90. 